\title{
Religious Affiliation and Ethics: Patterns Regarding Beliefs for Workplace Behaviour
}

\author{
Joshua Fogel \\ City University of New York, United States \\ Nadine McSween \\ Osman Dutt \\ Chartis, United States
}

\begin{abstract}
Ethical decisions are made regarding day-to-day workplace behaviours. We surveyed 315 college students from 9 different religious categories: Catholic, Christian, Jewish, Muslim, Hindu, Buddhist, Agnostic, Atheist, and Other. Individuals were asked whether "in the workplace, it is acceptable to do so" for 27 different behaviours. Those who were Hindu or Catholic consistently had scores indicating beliefs in performing more ethical behaviour. Those who were Buddhist or Atheist consistently had scores indicating beliefs in performing less ethical behaviour. In the analysis relating to 5 items regarding computer and Internet behaviours, those who were Jewish had scores indicating beliefs in performing less ethical behaviour. Managers who supervise young adults who recently graduated from college can be aware of possible ethical concerns facing those of different religions. They can mentor these individuals beginning their careers to adapt appropriate ethical behaviours for their job.
\end{abstract}

\section{Keywords}

Business ethics, religion, theology, work

\section{Introduction}

The 2007 National Business Ethics Survey in the United States found that 56\% of employees saw conduct at work that violated their company's ethics standards, policy, or the law. The greatest specific area (23\%) for this unethical behaviour was where employees placed their own interests above that of their company (Ethics Resource Center 2007). In a survey by the American Management Association, the top five areas influencing business ethics were corporate scandals, marketplace competition, demands by investors, pressure from customers, and globalization (American Management Association 2006).

A number of variables have been studied with regard to ethical behaviour at work. With regard to demographic variables, gender is often associated with ethical decision making. Although many

Copyright (C) 2010 Victoria University. This document has been published as part of the Journal of Business Systems, Governance and Ethics in both online and print formats. Educational and non-profit institutions are granted a nonexclusive licence to utilise this document in whole or in part for personal or classroom use without fee, provided that correct attribution and citation are made and this copyright statement is reproduced. Any other usage is prohibited without the express permission of the publisher. studies report that men are less ethical than women with regard to a number of business ethics topics, other studies do not report such a difference (McCabe et al. 2006). One suggestion for these differences in study outcomes is that besides gender there are social and psychological components for the construct of gender that need to be measured too (McCabe et al. 2006). 
Situational experiences can include the quality of work experience and are associated with ethical behaviour at work. For example, poor quality of work experience is associated with unethical behavioural intentions (Jones and Kavanagh 1996). Also, employee dissatisfaction is associated with increased unethical behaviour such as theft (Greenberg 1990; Greenberg 1993).

Personality characteristics also are associated with ethical behaviour at work. This includes the Protestant Work Ethic, an approach based upon ascetic Protestant values that includes devotion to work as a calling, hard work, autonomy, and thriftiness (Ghorpade et al. 2006). Those who score high on the Protestant Work Ethic report that questionable extra-organizational behaviours and questionable intra-organizational behaviours are less acceptable than those who score low on the Protestant Work Ethic (Jones et al. 2005).

\section{Literature Review for Religiosity and Ethical Behaviour at Work}

Besides the Protestant Work Ethic, there are studies on how religiosity influences ethics at work. Many of these studies report that greater religiosity is associated with greater ethical attitudes and behaviour in workplace settings. One study with a sample of 473 Christian business students from a number of denominations sought to determine how level of religiosity was associated with attitudes associated with corporate social responsibility. In the analyses for attitudes associated with ethical aspects of social responsibility, those from the high religiousness group had significantly greater concerns for the ethical aspects of corporate responsibility than those from the low religiousness group (Angelidis and Ibrahim 2004). In a different later study by these authors (Ibrahim et al., 2008) that included 506 students and 411 managers, that study sought to determine how level of religiosity was associated with attitudes associated with corporate social responsibility. A similar pattern as above occurred for the students with regard to the ethical aspects of corporate responsibility where those from the high religiousness group had significantly greater concerns for the ethical aspects of corporate responsibility than those from the low religiousness group. However for managers, there were no differences with regard to the ethical aspects of corporate responsibility between those from the high religiousness group and those from the low religiousness group. Students were also compared to managers in separate analyses for both those from low and high religiousness groups. In each of those separate analyses, whether comparing those with low religiousness or those with high religiousness, students had significantly greater concerns than managers for the ethical aspects of corporate responsibility.

Another study with a sample of 732 students used weekly church attendance as a measure of religiosity. This study sought to determine whether religiosity as measured by weekly church attendance was associated with ratings of acceptability for vignettes of ethically questionable behaviours. This study found that decreased religiosity levels as measured by decreased frequency of weekly church attendance was associated with increased ratings of acceptability for 7 of 25 vignettes of ethically questionable behaviours. These ethically questionable behaviours were underreporting income for taxes, bribing a foreign official, bribing purchase agents, using accounting tricks to conceal information, using deceptive advertising, violating software copyright issues, and bribing a manager to make a sale (Conroy and Emerson 2004).

Another study consisted of a sample of 490 students from an Evangelical college, Catholic college, and two public colleges. The study sought to determine whether attendance at religious affiliated colleges versus public colleges had an association with willingness to endorse unethical business behaviour. The study also sought to determine whether those who are more religious (defined as those more conservative, more fundamentalist, and intrinsically religious) were less likely to endorse unethical business behaviour as compared to those who were less religious. The study found that those from the Evangelical college were significantly less likely to endorse unethical business behaviour than those from the Catholic or public colleges. Also, those that were more religious from any of the colleges were significantly less likely to endorse unethical business behaviour than those who were less religious (Kennedy and Lawton 1998).

A study with a sample of 429 Muslim students from Malaysia sought to determine whether those who were more religious were less tolerant of unethical business practices than those who were less 
religious. The study also sought to determine whether an emphasis of study on Islamic studies rather than on secular studies such as Accounting or Business was associated with tolerance level for unethical business practices. The study found that those who were more religious were less tolerant of unethical business practices than those who were less religious. Also, individuals majoring in Islamic studies were less tolerant of unethical business practices than those majoring in secular studies. However, the study authors caution that the findings for those majoring in Islamic studies can be related to the fact that those majoring in Islamic studies had greater religiosity levels than those majoring in secular studies (Muhamad 2009).

In a sample of 20 senior executives from a number of religions, the study sought to determine whether religious belief was associated with awareness of encountering business ethical dilemmas. The study found that there were a number of religious beliefs that were associated with the perception of encountering business ethical dilemmas. This included a belief in monotheism, a transcendental standard of good and evil, the existence of heaven and hell, and high levels of praying and participation in the religious community (Graafland et al. 2006).

Although many of the studies above suggest a number of ways to measure religiosity as an important factor associated with practicing greater ethical business behaviour, a study published prior to 2000 does not exclusively show this pattern. A study of 1,224 managers in Hong Kong sought to determine whether religiosity was associated with ethical behaviour. The study found that there were significant differences for 9 of the 14 ethical dilemmas, where those who had a religious affiliation were less likely to agree with unethical activity than those without any religious affiliation. These 9 ethical dilemmas were deceptive gaining of competitor information, exposure of personal error, deceptive labeling and advertising, manipulation of expenses, nepotism, insider trading, sexual equity, bribery of third parties, and protection of dishonest employees. However, when importance of religious belief was compared rather than just religious affiliation, only 5 rather than 9 of the 14 ethical dilemmas were significantly different. In this analysis, those who believed in the importance of religious belief were less likely to agree with unethical activity than those who did not believe in the importance of religious belief. The study did not specify these particular 5 ethical dilemmas (McDonald and Pak 1997).

\section{Theoretical Basis}

The Hunt-Vitell theory of ethics was used as guidance for providing a theoretical basis for this study (Hunt and Vitell 2006). This theory helps explain how an individual faces a situation that the individual perceives as involving ethical concerns. The theory proposes different alternatives and actions that this individual can undertake to resolve the ethical dilemma. The two bases for the theory are that both the cultural environment and personal characteristics are important areas for how an individual perceives an ethical problem. Religion is very much emphasized as an integral part of this theory and is included as both part of the cultural environment and also as part of the personal characteristics categories. Many studies use this theory for both business ethics and general ethics topics (Hunt and Vitell 2006).

\section{Study Aims}

Religion is an important component related to ethical behaviour. We are not aware of any study that compares numerous religious groups simultaneously with regard to beliefs about ethical behaviour at work. Our primary aim is to understand the general beliefs and also to compare whether there are differences with regard to beliefs for ethical behaviour at work among those who are of Christian, Jewish, Muslim, Catholic, Hindu, Buddhist, Atheist, Agnostic, or Other religious affiliation. As part of this analytic approach we also adjust for religious observance status since being born into a faith does not necessarily indicate that one currently practices the tenets of that faith. An additional aim is to adjust for the potentially relevant variables that may affect this relationship including age, sex, race/ethnicity, having taken an ethics course, and being native born in the United States. 


\section{Method}

\section{Participants and Procedures}

Participants were 315 college students from Brooklyn College of the City University of New York. This is a commuter school where all the students live off campus and many live with their families. Of the 369 surveys distributed, 316 completed surveys were received. One individual omitted the religious affiliation category, so a total of 315 surveys were analysed. This is a response rate of $85.4 \%$ [(315/369)*100\%]. Participation in the survey was completely voluntary, with the sampling method being a convenience sample. Participants were approached to complete the surveys within their classrooms and in common areas of the college. Data were collected without any identifying information in an anonymous manner and occurred during March 2006. The survey was exempt from Institutional Review Board review and was conducted consistent with the ethical standards of the Declaration of Helsinki. Informed consent was provided.

\section{Demographics Items}

Items included age measured in years, sex (man/woman), race/ethnicity, took ethics course (no/yes), and born in the United States (no/yes).

\section{Religiosity Items}

The independent variable was, "What is your religious status?" with 9 choices of Christian, Jewish, Muslim, Catholic, Hindu, Buddhist, Atheist, Agnostic, or Other. An additional question regarding religious observance level used as a covariate was, "Would you describe yourself as religious?" with choices of no/yes.

\section{Ethical Behaviour at Work Belief Items}

Participants were asked to answer twenty-seven questions relating to their beliefs about the ethical acceptability of certain workplace behaviours. The stem portion was, "In the workplace, it is acceptable to:" and measured with a Likert-style scale with $1=$ strongly disagree to $5=$ strongly agree. For the items used, strongly disagree indicates more ethical behaviour and strongly agree indicates more unethical behaviour. These items included: "take merchandise/equipment for one's own personal use," "do less than your share of work in a group project," "use unethical behaviour to earn a promotion/gain a sale, "take money from the company," and "receive non-work related emails." The first 21 items were used from the survey conducted by Nonis and Swift (2001) while the other 6 items with 5 focusing on Internet topics and 1 general item were created specifically for this study.

\section{Statistical Analysis}

Descriptive statistics were used to describe the sample characteristics and general attitude levels. Also, an overall ranking of items was done to describe patterns. Inferential statistical analyses were conducted with the independent variable of the religious status variable with the 9 choices. The dependent variables were the 27 ethical items. First a multivariate analysis of covariance (MANCOVA) was conducted with the dichotomous religious observance level variable as the covariate. Then, three separate models were analysed for each of the 27 ethical items. The first model was analysed with analysis of variance (ANOVA) and included just the nine-level religious status variable. The second model was analysed with analysis of covariance (ANCOVA) and included the dichotomous religious observance level variable as a covariate. The third model was analysed with ANCOVA and included the religious level variable and also age, sex, race/ethnicity, took ethics course, and born in the United States. Also, the items that had overall statistical significance in the three models above were analysed with the same analytic approach among the subset of individuals with either part-time or full-time employment. SPSS Version 16.0.2 was used for all analyses (SPSS 2008). 


\section{Results}

Table 1 shows the demographic characteristics. Average age was more than 22 years. There were approximately equal numbers of men and women. (Note: $\mathrm{M}=$ mean, $\mathrm{SD}=$ standard deviation.)

\begin{tabular}{|l|l|l|}
\hline Variable & $\%(\mathrm{n})$ & $\mathrm{M}(\mathrm{SD})$ \\
\hline Age & & $22.78(5.10)$ \\
\hline Sex & $45.1 \%(142)$ & \\
Men & $54.9 \%(173)$ & \\
Women & & \\
\hline Race/ethnicity & $18.7 \%(59)$ & \\
African-American & $11.7 \%(37)$ & \\
Hispanic-American & $10.2 \%(32)$ & \\
Asian/Asian-American & $13.3 \%(42)$ & \\
Southeast Asian & $35.9 \%(113)$ & \\
White & $10.2 \%(32)$ & \\
Other & & \\
\hline Ethics Course & $51.4 \%(162)$ & \\
No & $48.6 \%(153)$ & \\
Yes & & \\
\hline Born in US & $51.4 \%(162)$ & \\
No & $48.6 \%(153)$ & \\
Yes & & \\
\hline Employment Status & $21.3 \%(67)$ & \\
Full-time & $55.9 \%(176)$ & \\
Part-time & $22.9 \%(72)$ & \\
Not Working & & \\
\hline Religious Status & $26.3 \%(83)$ & \\
Christian & $9.5 \%(30)$ & \\
Jewish & $12.7 \%(40)$ & \\
Muslim & $26.3 \%(83)$ & \\
Catholic & $2.9 \%(9)$ & \\
Hindu & $3.8 \%(12)$ & \\
Buddhist & $3.8 \%(12)$ & \\
Agnostic & $3.2 \%(10)$ & \\
Atheist & $11.4 \%(36)$ & \\
Other & & \\
\hline Religious & $52.7 \%(166)$ & \\
No & $47.3 \%(149)$ & \\
Yes & \\
\hline
\end{tabular}

Table 1: Characteristics of the Sample of 315 Individuals

There was diverse racial/ethnic representation with almost 20\% African American, and the Hispanic, Asian/Asian American, and Southeast Asian categories with at least 10\% for each. Whites comprised only a bit more than one-third of the sample. Almost half had taken an ethics course. Slightly more than half were born outside of the United States. With regard to employment, more than half worked part-time and more than $20 \%$ worked full-time. With regard to religious status, more than one-quarter were Christian, more than one-quarter were Catholic, more than $10 \%$ were Muslim, and almost $10 \%$ were Jewish. Almost half of the sample reported being religious.

The top three rankings for lowest scores (more ethical beliefs) for mean values for all 27 items (see Table 2) were counted. Hindu had the greatest number with 21, Other with 17 (with one tie) was next highest, followed by Catholic with 16 (with one tie). The other counts included Agnostic with 12 (with one tie), Muslim with 8, Jewish with 3, Atheist with 3, Christian with 2 (with one tie), and Buddhist with 1. In the subset of the 5 items (\#22 - \#26) relating to computers and the Internet and the top three rankings for lowest scores (more ethical beliefs), Hindu and Agnostic were included for all 5 items, Catholic with 3 items, and Muslim with 2 items. No other religious groups were ranked for these items. 
The top three rankings for highest scores (less ethical beliefs) for mean values for all 27 items (see Table 2) were counted. Buddhist had the greatest number with 24 (with two ties) and Atheist with 18 (with one tie) was next highest. The next few rankings had similar counts and consisted of Jewish with 11, Muslim with 10, and Agnostic with 10 (with one tie). The rest of the rankings were Christian with 6 (with one tie), Hindu with 3, Catholic with 1, and Other with 1. In the subset of the 5 items (\#22 \#26) relating to computers and the Internet, Jewish had high score rankings for all 5 items, both Buddhist and Atheist had high score rankings with 4 items, and Christian with 3 items. This ranking included one tie for Atheist and Christian. No other religious groups had high score rankings for these items.

In the inferential statistical analyses, the Wilks' lambda MANCOVA omnibus test with religious category as the independent variable and adjusting for religious observance status was significant $(\mathrm{p}=0.048)$, and the null hypothesis that the 27 ethics items did not differ was rejected. The separate ANOVA analyses (see Table 2) indicated overall statistical significance or approaching significance for the 5 items of "take merchandise/equipment for one's own personal use (\#3)," "complete personal business on company time (\#4)," "do less than your share of work in a group project (\#8)," "take money from the company (\#21)," and "purchase items online (\#25)." In the ANCOVA analysis adjusting for the religious observance level variable, the same pattern of significance or approaching significance occurred (see Figures 1 through 5). In the ANCOVA analysis adjusting for numerous relevant covariates, the same pattern of significance or approaching significance occurred except for "take money from the company" which now had a p-value of 0.102 (see Figures 1 through 5). 


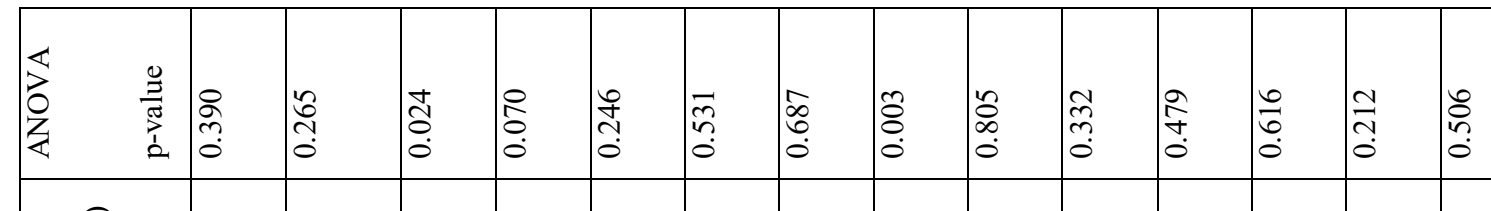

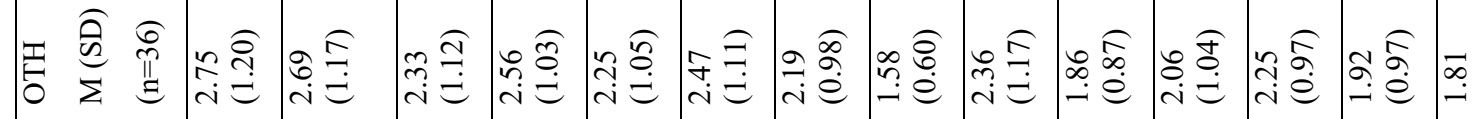

तิ

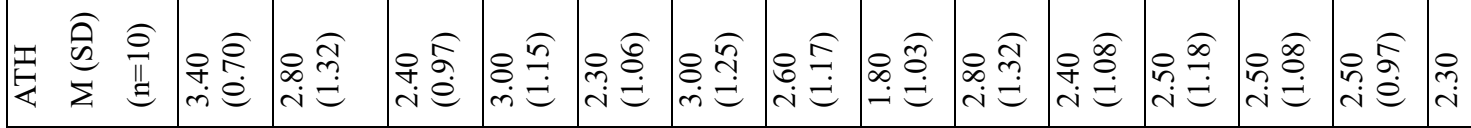

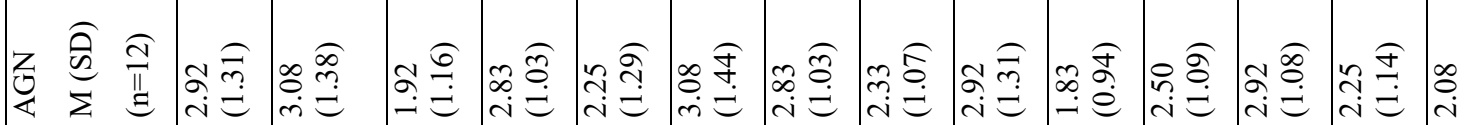

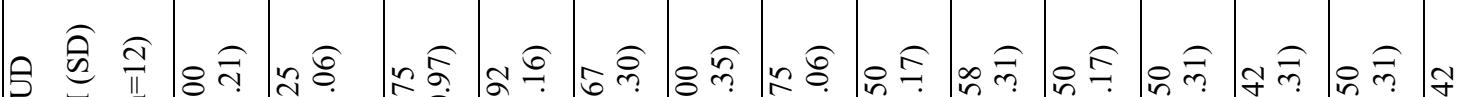

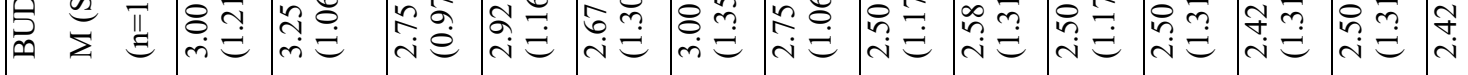

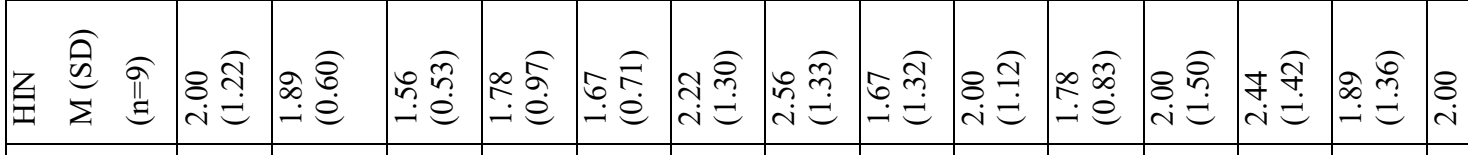

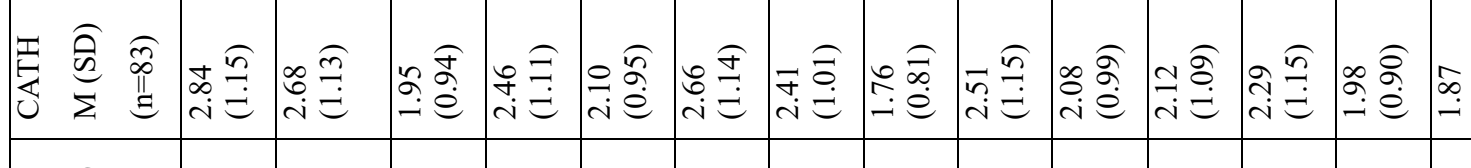

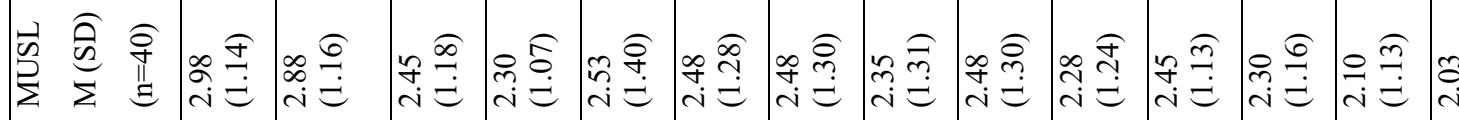

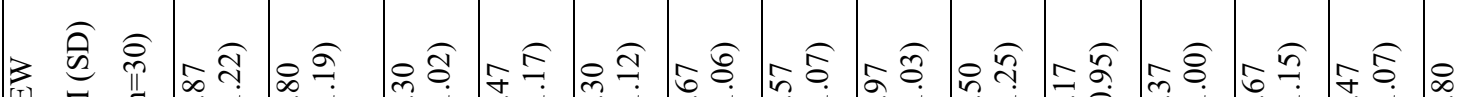

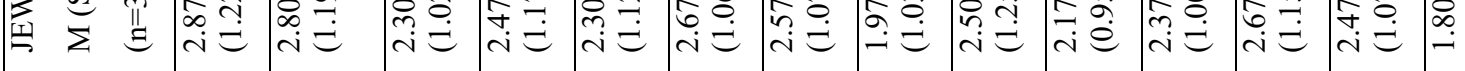

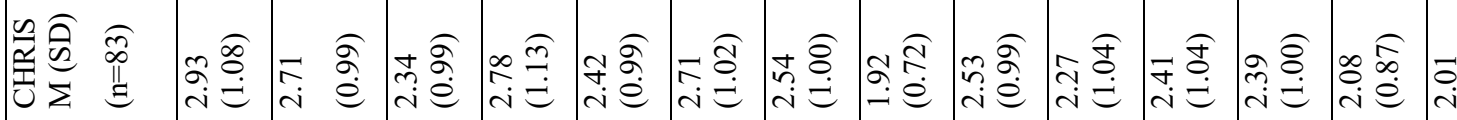

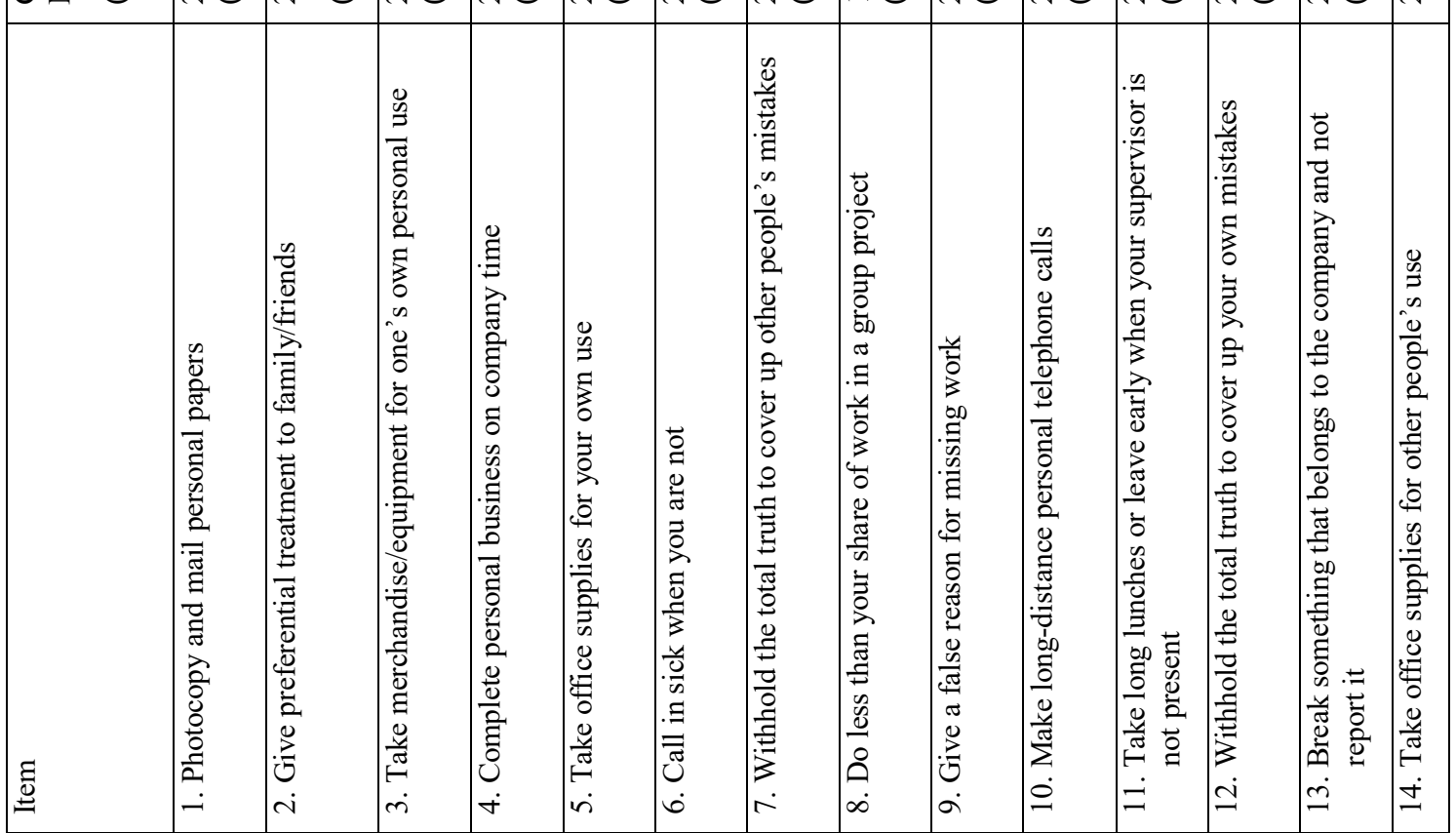




\begin{tabular}{|c|c|c|c|c|c|c|c|c|c|c|c|c|c|c|}
\hline & $\begin{array}{l}\infty \\
0 \\
\stackrel{0}{0} \\
0\end{array}$ & đ̂̃ & $\tilde{n}$ & $\frac{\text { P }}{\stackrel{9}{0}}$ & \begin{tabular}{l}
$\forall$ \\
\multirow{2}{*}{} \\
0 \\
0
\end{tabular} & 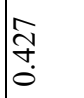 & $\begin{array}{l}\mathscr{O} \\
0 \\
0 \\
0\end{array}$ & $\frac{n}{2}$ & $\frac{m}{\ddot{n}}$ & $\underset{f}{\mathscr{f}}$ & $\begin{array}{l}\stackrel{ \pm}{0} \\
0 \\
0\end{array}$ & $\frac{\vec{\infty}}{\overrightarrow{0}}$ & $\frac{\infty}{\stackrel{\infty}{\eta}}$ & \\
\hline बิ & 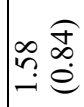 & 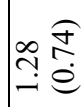 & 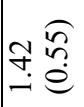 & 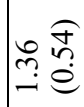 & 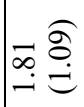 & શે & $\hat{i} \underset{\hat{\sigma}}{\hat{\sigma}}$ & $\stackrel{\overbrace{}}{\stackrel{n}{i}} \stackrel{\overbrace{}}{=}$ & 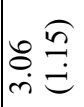 & $=\stackrel{E}{=}$ & 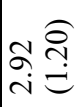 & 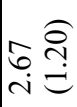 & 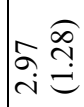 & \\
\hline $\begin{array}{c}\overparen{\hat{\sigma}} \\
\infty \\
\dot{e}\end{array}$ & $\stackrel{\overbrace{}}{\approx 0}$ & 옹 & 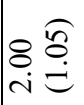 & 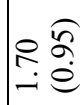 & 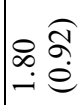 & 吾 & $\stackrel{\overparen{\overbrace{}}}{\stackrel{\overbrace{}}{\smile}}$ & 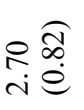 & $\begin{array}{ll}0 & \infty \\
0 & \infty \\
\dot{m} & \stackrel{0}{e}\end{array}$ & 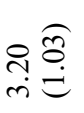 & 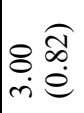 & 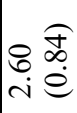 & 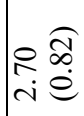 & \\
\hline $\begin{array}{l}\hat{\S} \\
\hat{\varrho} \\
\hat{e}\end{array}$ & $\begin{array}{ll}\infty & \widehat{\theta} \\
0 & 0 \\
i & 0\end{array}$ & $\stackrel{\overbrace{}}{\stackrel{2}{9}}$ & 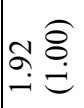 & 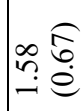 & 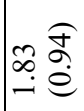 & 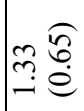 & 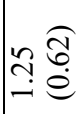 & 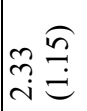 & 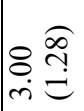 & 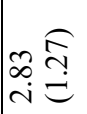 & 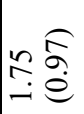 & 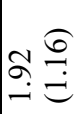 & 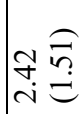 & \\
\hline $\begin{array}{l}\underline{6} \\
\stackrel{-}{\beth}\end{array}$ & 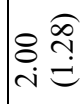 & 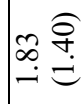 & 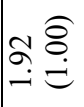 & 突 & 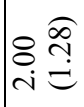 & $\underline{\sigma} \underset{\sigma}{\sigma}$ & $\widehat{\sigma} \widehat{\widehat{\sigma}}$ & 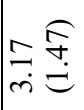 & 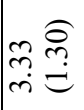 & $\begin{array}{l}\stackrel{\overparen{f}}{\stackrel{f}{\rightleftarrows}} \\
\dot{m}\end{array}$ & 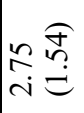 & 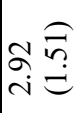 & 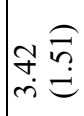 & 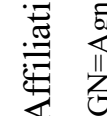 \\
\hline$\stackrel{\overparen{\overbrace{}}}{\stackrel{\overbrace{}}{\Xi}}$ & 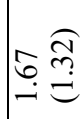 & $\stackrel{\overbrace =}{\stackrel{f}{\rightleftarrows}}$ & 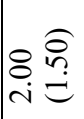 & 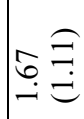 & 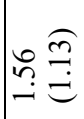 & 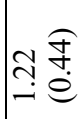 & \& & 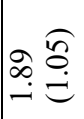 & $\begin{array}{l}\infty \hat{a} \\
\stackrel{-}{e}\end{array}$ & $=\stackrel{\overparen{\Im}}{\stackrel{\Im}{巳}}$ & 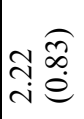 & 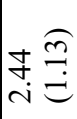 & 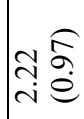 & 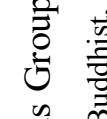 \\
\hline ई人̊ & 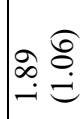 & \begin{tabular}{ll}
0 & $\widehat{\sigma}$ \\
\multirow{2}{*}{} & $\infty$ \\
- & 0
\end{tabular} & 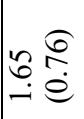 & 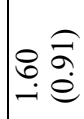 & 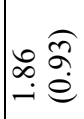 & 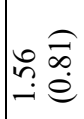 & 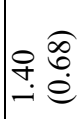 & 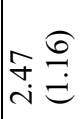 & 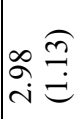 & 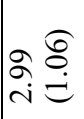 & 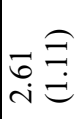 & 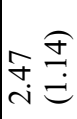 & 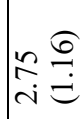 & $\begin{array}{l}\frac{0}{00} \\
\frac{0}{0} \\
\tilde{0}\end{array}$ \\
\hline$\stackrel{\overbrace{}}{\tilde{\sigma}}$ & 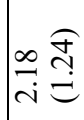 & 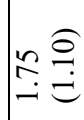 & 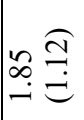 & 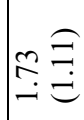 & 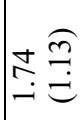 & 울 & 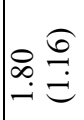 & $\begin{array}{ll}\circ & \widehat{n} \\
+ & \hat{a} \\
ن & \stackrel{e}{e}\end{array}$ & $\begin{array}{l}\hat{\sigma} \hat{a} \\
\dot{m}\end{array}$ & 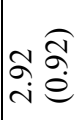 & $\stackrel{\widehat{\hat{n}}}{\stackrel{\sigma}{\ominus}}$ & 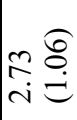 & 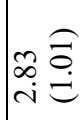 & $\begin{array}{l}\bar{\Xi} \\
\text { בै } \\
\pm \\
\Xi\end{array}$ \\
\hline $\begin{array}{l}\widetilde{\sigma} \\
\infty \\
\dot{e}\end{array}$ & 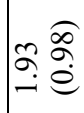 & 亲 & 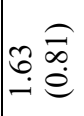 & 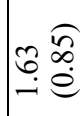 & 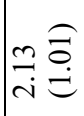 & $\begin{array}{ll}\stackrel{\overbrace{}}{\infty} \\
\stackrel{\infty}{e}\end{array}$ & $\begin{array}{ll}\stackrel{0}{\infty} \\
\stackrel{\infty}{e}\end{array}$ & 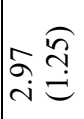 & 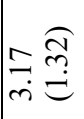 & 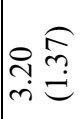 & $\hat{a} \stackrel{\widehat{a}}{i}$ & 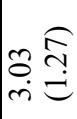 & $\begin{array}{l}0 \overparen{\pi} \\
\dot{m} \\
\dot{m}\end{array}$ & $\begin{array}{l}\stackrel{0}{0} \\
\stackrel{0}{\infty} \\
\vdots\end{array}$ \\
\hline $\begin{array}{l}\hat{\partial} \\
\dot{\infty} \\
\hat{e}\end{array}$ & 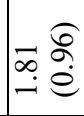 & 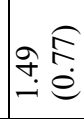 & $\begin{array}{ll}0 & 0 \\
0 & \circ \\
- & 0\end{array}$ & 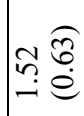 & 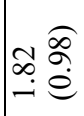 & 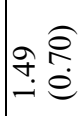 & 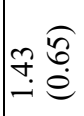 & 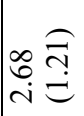 & $\begin{array}{l}\circ \stackrel{\infty}{=} \\
\dot{m}\end{array}$ & 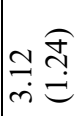 & 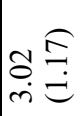 & 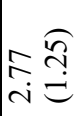 & 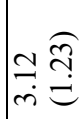 & 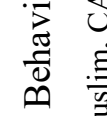 \\
\hline & 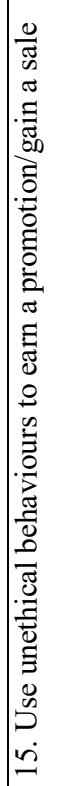 & 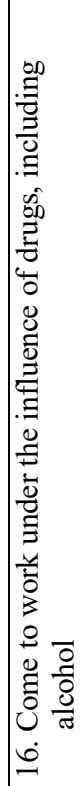 & 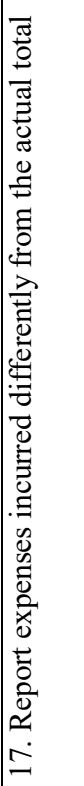 & 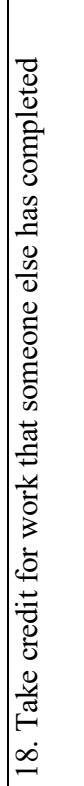 & 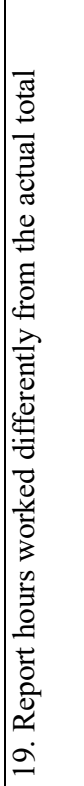 & 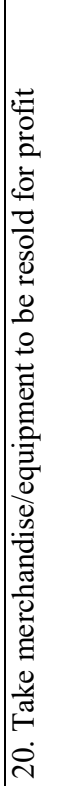 & 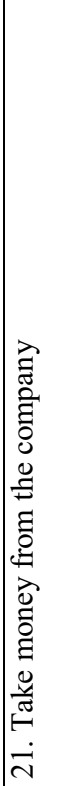 & 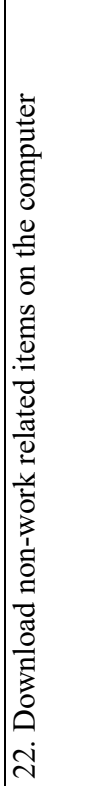 & 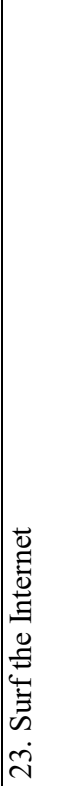 & 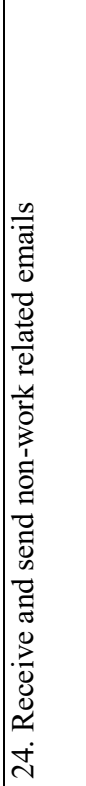 & 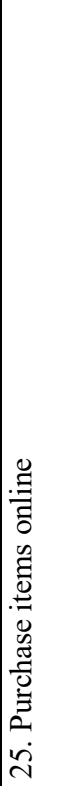 & 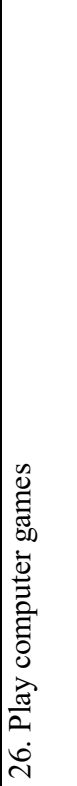 & 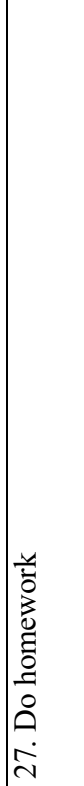 & 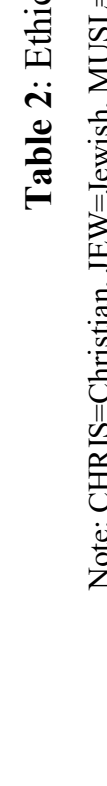 \\
\hline
\end{tabular}




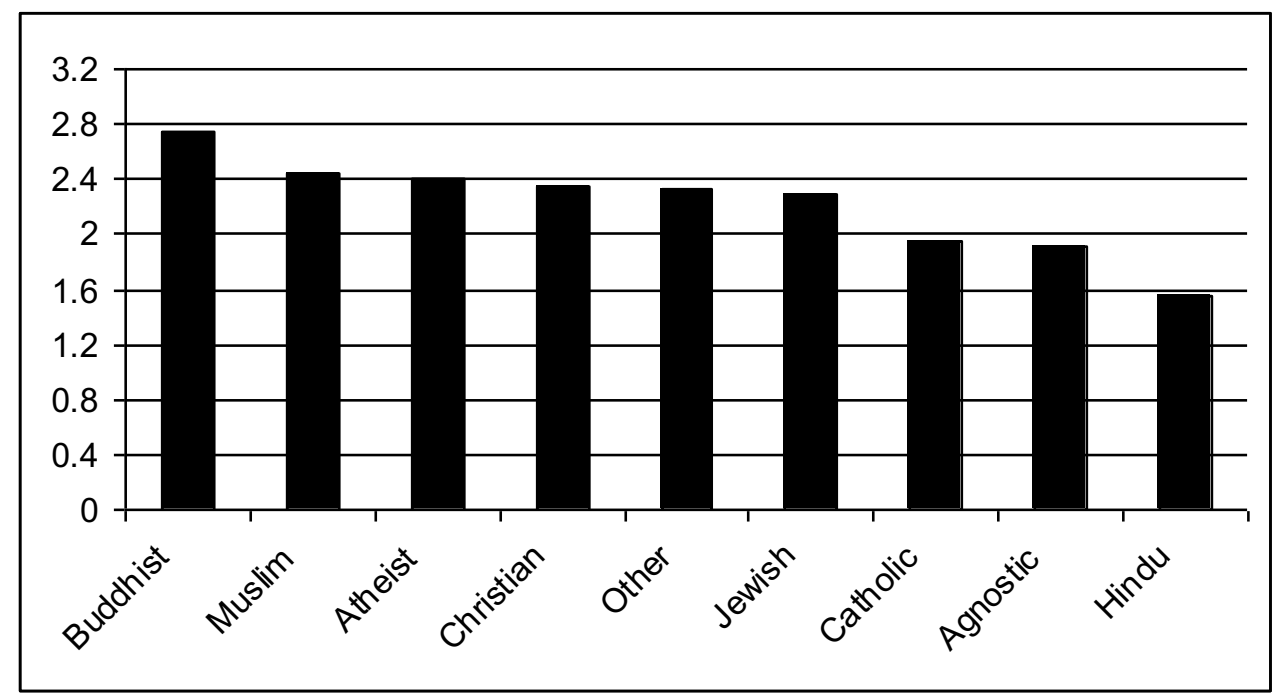

Figure 1: Mean Values for Take Merchandise/Equipment for One's Own Personal Use

Note: ANOVA Model 1: $\mathrm{p}=0.024$; ANCOVA Model 2: $\mathrm{p}=0.026$; ANCOVA Model 3: $\mathrm{p}=0.028$.

Measured with a Likert-style scale with $1=$ strongly disagree to $5=$ strongly agree

For these 5 items that were significant or approaching significance, the top three rankings for lowest scores (more ethical beliefs) for these 5 items were Hindu and Catholic, as both had the lowest ranks with 5 items. The other groups included Agnostic with 3 items, Muslim with 1 item, and Other with 1 item. With regard to the top three rankings for highest scores (least ethical beliefs), Atheist and Buddhist (with one tie) were both highest with 4 items. The other groups included Muslim with 3 items, Agnostic with 2 items, and Christian, Jewish, and Other all with 1 item.

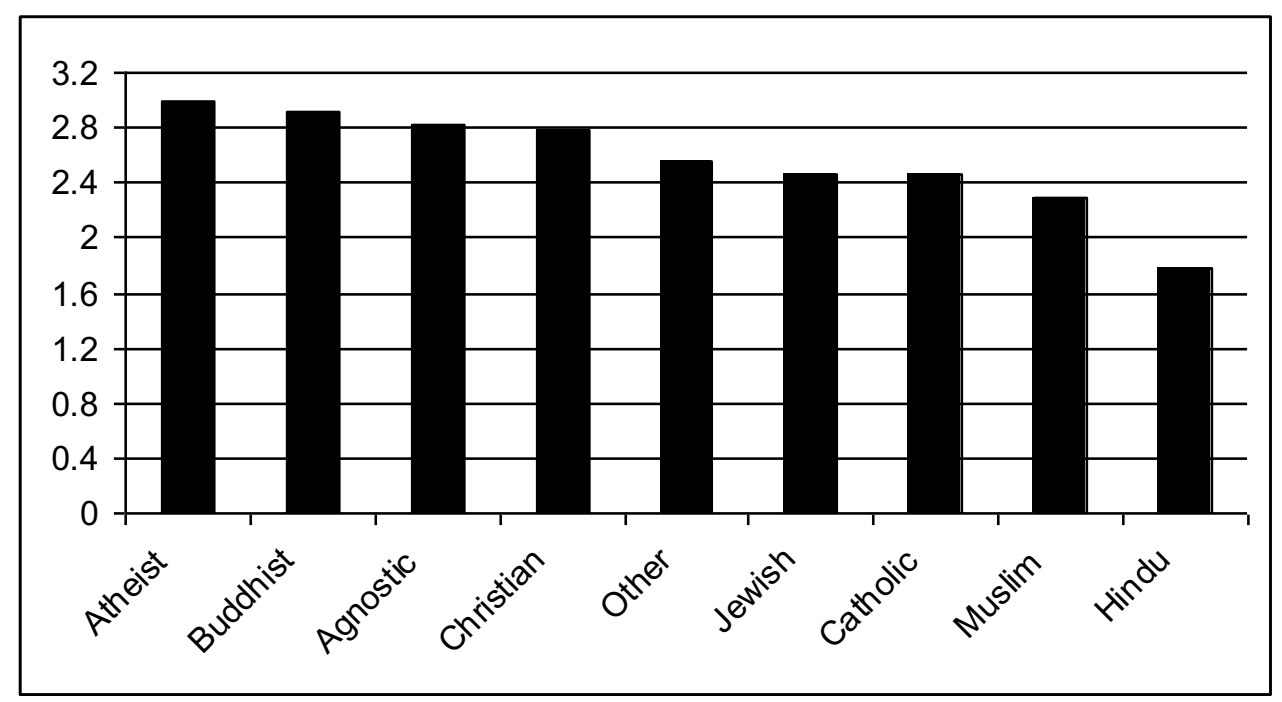

Figure 2: Mean Values for Complete Personal Business on Company Time

Note: ANOVA Model 1: $\mathrm{p}=0.070$; ANCOVA Model 2: $\mathrm{p}=0.075$; ANCOVA Model 3: $\mathrm{p}=0.082$.

Measured with a Likert-style scale with $1=$ strongly disagree to $5=$ strongly agree 


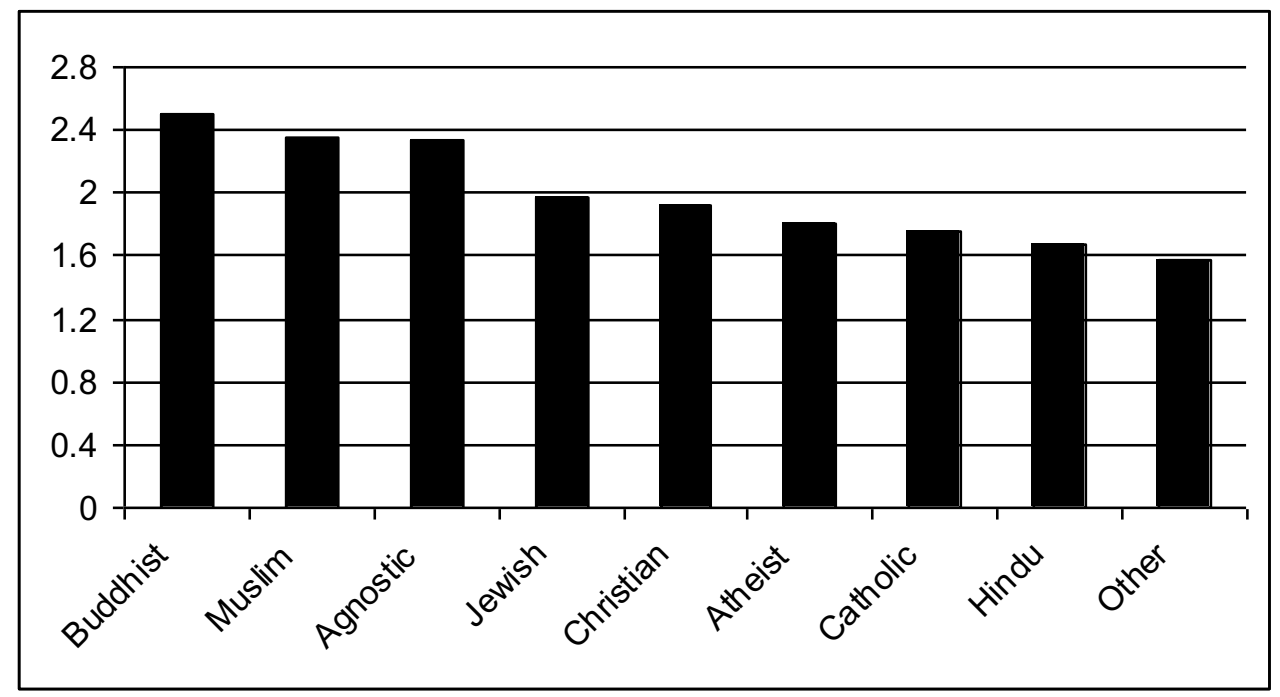

Figure 3: Mean Values for Do Less than Your Share of Work in a Group Project

Note: ANOVA Model 1: $\mathrm{p}=0.003$; ANCOVA Model 2: $\mathrm{p}=0.003$; ANCOVA Model 3: $\mathrm{p}=0.005$.

Measured with a Likert-style scale with $1=$ strongly disagree to $5=$ strongly agree

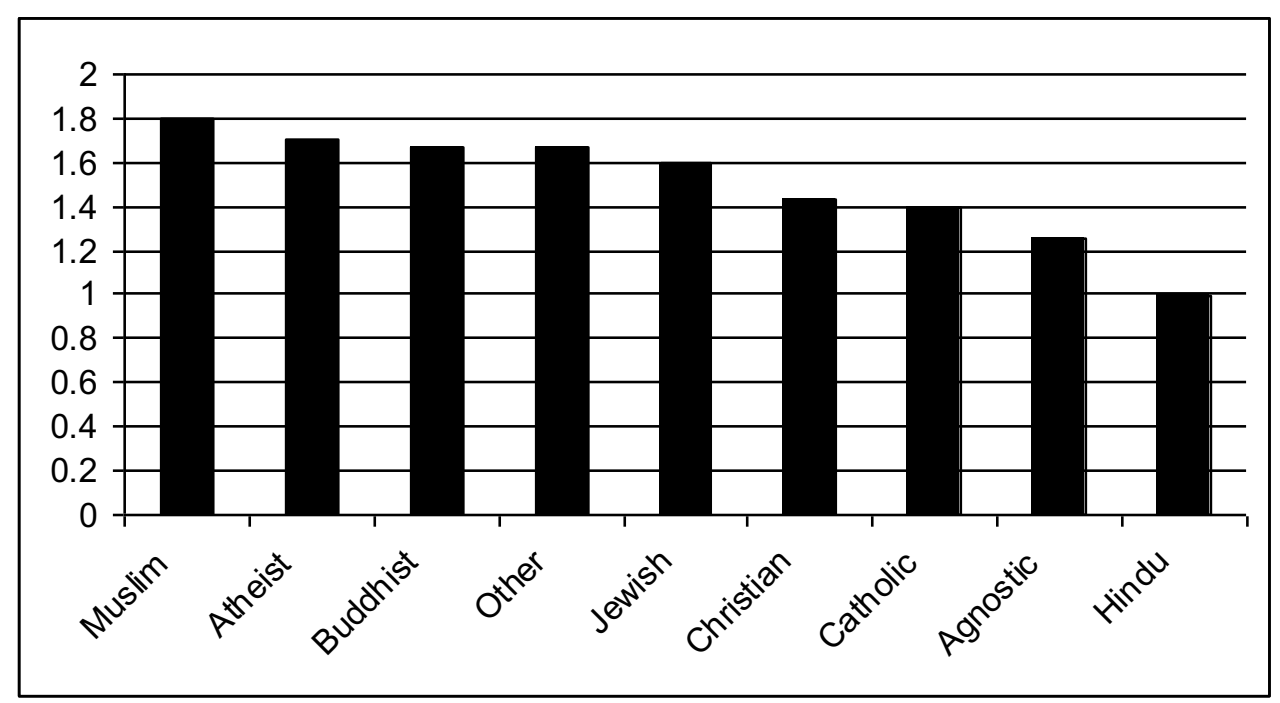

Figure 4: Mean Values for Take Money from the Company

Note: ANOVA Model 1: $\mathrm{p}=0.083$; ANCOVA Model 2: $\mathrm{p}=0.082$; ANCOVA Model 3: $\mathrm{p}=0.102$.

Measured with a Likert-style scale with $1=$ strongly disagree to $5=$ strongly agree 


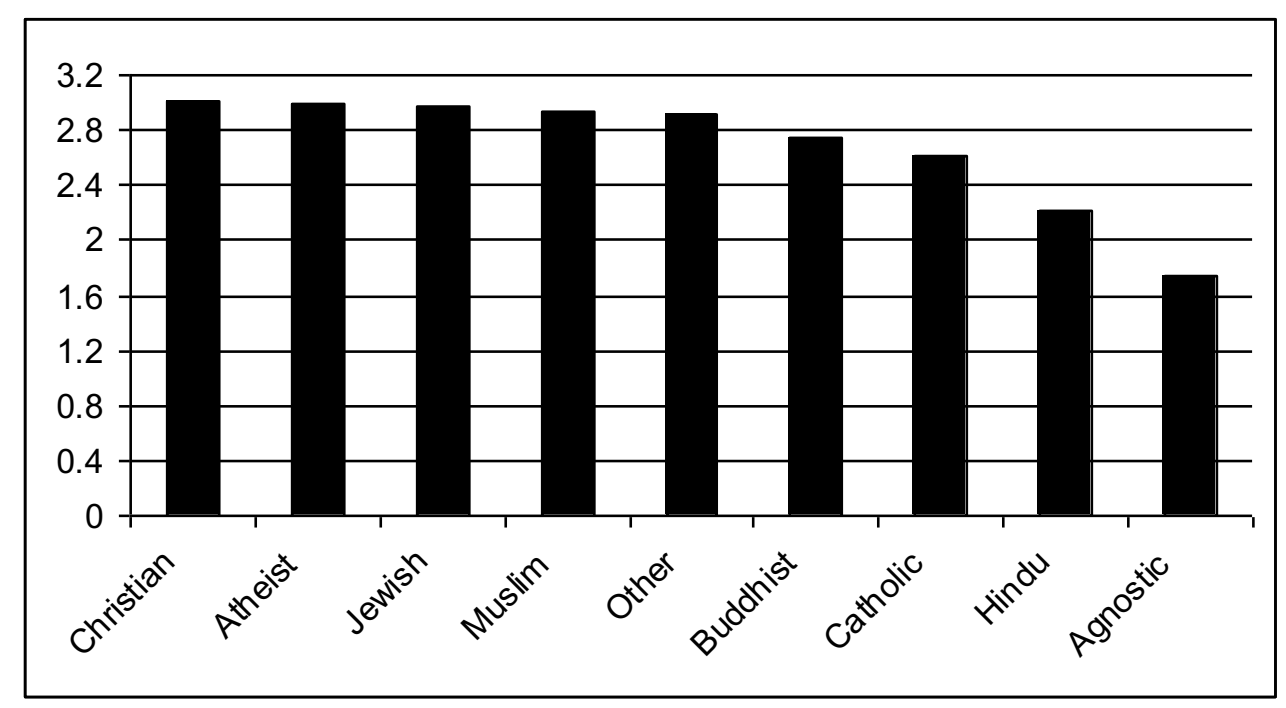

Figure 5: Mean Values for Purchase Items Online

Note: ANOVA Model 1: $p=0.014$; ANCOVA Model 2: $p=0.014$; ANCOVA Model 3: $p=0.007$. Measured with a Likert-style scale with $1=$ strongly disagree to $5=$ strongly agree

Post-hoc least significance difference tests for these 5 items were as follows. For "take merchandise/equipment for one's own personal use," Hindu had the lowest mean and significantly differed from Christian, Muslim, Buddhist, and Other. Also, Hindu approached significance to differ from Jewish $(\mathrm{p}=0.055)$ and Atheist $(\mathrm{p}=0.072)$. Although Agnostic had the next lowest mean, it only significantly differed from Buddhist. Catholic had the third lowest mean and significantly differed from Christian, Muslim, and Buddhist and approached significance to differ from Other ( $\mathrm{p}=0.061)$.

For "complete personal business on company time," Hindu had the lowest mean and significantly differed from Christian, Buddhist, Agnostic, and Atheist. Hindu also approached significance to differ from Catholic $(p=0.080)$ and Other $(p=0.059)$. Muslim had the next lowest mean and significantly differed from Christian and approached significance to differ from Buddhist $(\mathrm{p}=0.090)$ and Atheist $(p=0.073)$. Catholic approached significance to differ with a lower mean than Christians $(p=0.058)$.

For "do less than your share of work in a group project," Other had the lowest mean and significantly differed from Muslim, Buddhist, and Agnostic. Other also approached significance to differ from Christian $(\mathrm{p}=0.071)$ and Jewish $(\mathrm{p}=0.092)$. Hindu had the second lowest mean and significantly differed from Muslim and Buddhist. Catholic had the third lowest mean and significantly differed from Muslim, Buddhist, and Agnostic. Atheist had the fourth lowest mean and approached significance to differ from Muslim ( $\mathrm{p}=0.091)$ and Buddhist $(\mathrm{p}=0.076)$. Christian significantly differed with lower means than Muslim and Buddhist. Jewish approached significance with lower means to differ from Muslim ( $\mathrm{p}=0.085)$ and Buddhist $(\mathrm{p}=0.090)$.

For "take money from the company," Hindu had the lowest mean and significantly differed from Muslim and Other. Hindu also approached significance to differ from Jewish $(\mathrm{p}=0.059)$, Buddhist $(\mathrm{p}=0.071)$, and Atheist $(\mathrm{p}=0.069)$. Muslim had the highest mean and significantly differed from Christian, Catholic, Hindu, and Agnostic.

For "purchase items online," Agnostic had the lowest mean and significantly differed from all the 8 groups except not for Hindu. Hindu had the second lowest mean and significantly differed from Christian. Hindu also approached significance to differ from Jewish $(\mathrm{p}=0.088)$ and Muslim $(\mathrm{p}=0.097)$. Catholic had the third lowest mean and significantly differed from Christian and Agnostic.

Besides the 5 items that had overall statistical significance, all 22 other items had either statistical significance or approached significance for post-hoc tests between the 9 different religious categories. For "photocopy and mail personal papers (\#1)," Hindu had the lowest mean and significantly differed from all groups except for where it approached significance for Agnostic $(p=0.070)$ and Other $(\mathrm{p}=0.080)$. For "give preferential treatment to family/friends $(\# 2)$," Hindu had the lowest mean and 
significantly differed from all groups except for where it approached significance for Atheist $(p=0.075)$ and Other $(p=0.053)$. For "take office supplies for your own use (\#5)," Hindu had the lowest mean and significantly differed from Christian, Muslim, and Buddhist. Catholic had the next lowest mean and significantly differed from Muslim. Catholic also approached significance for Christian ( $\mathrm{p}=0.053)$ and Buddhist $(\mathrm{p}=0.088)$. For "call in sick when you are not (\#6)," Hindu had the lowest mean and approached significance to differ from Agnostic $(\mathrm{p}=0.089)$. For "withhold the total truth to cover up other people's mistakes (\#7)," Other had the lowest mean and significantly differed from Agnostic ( $\mathrm{p}=0.074)$.

For "give a false reason for missing work (\#9)," Hindu had the lowest mean and approached significance to differ from Agnostic ( $\mathrm{p}=0.074)$. For "make long-distance personal telephone calls (\#10)," Other had the third lowest mean and significantly differed from Christian and also approached significance to differ from Muslim ( $\mathrm{p}=0.080)$ and Buddhist $(\mathrm{p}=0.063)$. For "take long lunches or leave early when your supervisor is not present (\#11)," Catholic had the third lowest mean and approached significance to differ with a lower mean than Christian ( $\mathrm{p}=0.089)$. For "withhold the total truth to cover up your own mistakes (\#12)," Agnostic had the highest mean and approached significance to differ from Muslim $(\mathrm{p}=0.091)$, Catholic $(\mathrm{p}=0.067)$, and Other $(\mathrm{p}=0.071)$. For "break something that belongs to the company and not report it (\#13)," Hindu had the lowest mean, Other had the second lowest mean and significantly differed from Jewish and also approached significance to differ from Buddhist $(\mathrm{p}=0.079)$. Catholic had the third lowest mean and significantly differed from Jewish and approached significance to differ from Buddhist $(\mathrm{p}=0.088)$. Christian had the fourth lowest mean and approached significance to differ from Jewish $(p=0.071)$. For "take office supplies for other people's use (\#14)," Jewish had the lowest mean and approached significance to differ from Buddhist $(\mathrm{p}=0.058)$. Other had the second lowest mean and approached significance to differ from Buddhist $(p=0.054)$. Catholic had the third lowest mean and approached significance to differ from Buddhist $(\mathrm{p}=0.062)$.

For "use unethical behaviours to earn a promotion/gain a sale (\#15)," Other had the lowest mean and significantly differed from Muslim. Muslim had the highest mean and approached significance to differ from Christian ( $\mathrm{p}=0.068$ ). For "come to work under the influence of drugs, including alcohol (\#16)," Other had the lowest mean and significantly differed from Muslim and also approached significance to differ from Hindu $(\mathrm{p}=0.067)$ and Buddhist $(\mathrm{p}=0.062)$. Catholic had a lower mean that approached significance to differ from Muslim $(\mathrm{p}=0.090)$. For "report expenses incurred differently from the actual total (\#17)," Other had the lowest mean and significantly differed from Muslim and also approached significance to differ from Hindu $(p=0.085)$, Buddhist $(p=0.098)$, Agnostic $(p=0.098)$, and Atheist ( $\mathrm{p}=0.072)$. For "take credit for work that someone else has completed (\#18)," Other had the lowest mean and approached significance to differ from Muslim $(\mathrm{p}=0.056)$. For "report hours worked differently from the actual total (\#19)," Muslim had a lower mean that approached significance to differ from Jewish ( $\mathrm{p}=0.097$ ). For "take merchandise / equipment to be resold for profit (\#20)," Hindu had the lowest mean and approached significance to differ from Muslim ( $p=0.075)$. Agnostic had the next lowest mean. Other had the third lowest mean and approached significance to differ from Muslim ( $\mathrm{p}=0.051)$. Christian had the fourth lowest mean and approached significance to differ from Muslim ( $\mathrm{p}=0.098$ ). For "do homework (\#27)," Hindu had the lowest mean and significantly differed from Christian, Buddhist and also approached significance to differ from Jewish $(\mathrm{p}=0.057)$ and Other $(\mathrm{p}=0.096)$. Agnostic had the second lowest mean and significantly differed from Agnostic and also approached significance to differ from Christian $(\mathrm{p}=0.060)$ and Jewish $(\mathrm{p}=0.098)$. Atheist had the third lowest mean. Catholic had the fourth lowest mean and significantly differed from Christian and also approached significance to differ from Buddhist $(\mathrm{p}=0.073)$.

For the computer and Internet items, for "download non-work related items on the computer (\#22)," Hindu had the lowest mean and significantly differed from Jewish and Buddhist and also approached significance to differ from Christian $(\mathrm{p}=0.056)$. Muslim had the next lowest mean and significantly differed from Jewish and Buddhist. Catholic had the third lowest mean and significantly differed from Jewish and also approached significance to differ from Buddhist $(\mathrm{p}=0.054)$. Buddhist had the highest mean and besides the differences above also approached significance to differ from Agnostic $(p=0.082)$ and Other $(p=0.088)$. For "surf the Internet $(\# 23)$," Hindu had the lowest mean and 
significantly differed from all 8 other groups. For "receive and send non-work related emails (\#24)," Hindu had the lowest mean and significantly differed from all the religious groups except for only approaching significance to differ from Muslim $(\mathrm{p}=0.061)$ and did not differ from Agnostic. For "play computer games (\#26)," Agnostic had the lowest mean and significantly differed from Christian, Jewish, Muslim, Buddhist and also approached significance to differ from Other $(\mathrm{p}=0.059)$. Hindu had the second lowest mean. Catholic had the third lowest mean and significantly differed from Jewish.

\section{Analyses for those Employed Part-time or Full-time}

Additional analyses were performed for the subset of those who were employed part-time or full-time. Four of the 5 items that had overall significance remained significant with ANOVA (see Table 3). These items were "take merchandise/equipment for one's own personal use (\#3)," "complete personal business on company time (\#4)," "do less than your share of work in a group project (\#8)," and "purchase items online (\#25). However, "take money from the company (\#21)," no longer had overall statistical significance.

\begin{tabular}{|c|c|c|c|c|c|c|c|c|c|c|}
\hline Item & $\begin{array}{l}\text { CHRIS } \\
\text { M (SD) } \\
(\mathrm{n}=69)\end{array}$ & $\begin{array}{l}\text { JEW } \\
\mathrm{M} \\
(\mathrm{SD}) \\
(\mathrm{n}=25)\end{array}$ & $\begin{array}{l}\text { MUSL } \\
\text { M } \\
(\mathrm{SD}) \\
(\mathrm{n}=24)\end{array}$ & $\begin{array}{l}\text { CATH } \\
\text { M } \\
(\mathrm{SD}) \\
(\mathrm{n}=71)\end{array}$ & $\begin{array}{l}\text { HIN } \\
M \\
(\mathrm{SD}) \\
(\mathrm{n}=5)\end{array}$ & $\begin{array}{l}\text { BUD } \\
\text { M } \\
(\mathrm{SD}) \\
(\mathrm{n}=10)\end{array}$ & $\begin{array}{l}\text { AGN } \\
\text { M } \\
(\mathrm{SD}) \\
(\mathrm{n}=9)\end{array}$ & $\begin{array}{l}\text { ATH } \\
\text { M } \\
(\mathrm{SD}) \\
(\mathrm{n}=6)\end{array}$ & $\begin{array}{l}\text { OTH } \\
\text { M } \\
(\mathrm{SD}) \\
(\mathrm{n}=24)\end{array}$ & $\begin{array}{l}\text { ANOVA } \\
\text { p-value }\end{array}$ \\
\hline $\begin{array}{l}\text { 3. Take merchandise/equipment } \\
\text { for one's own personal use }\end{array}$ & $\begin{array}{l}2.33 \\
(1.01)\end{array}$ & $\begin{array}{l}2.32 \\
(1.11)\end{array}$ & $\begin{array}{l}2.50 \\
(1.18)\end{array}$ & $\begin{array}{l}1.97 \\
(0.96)\end{array}$ & $\begin{array}{l}1.20 \\
(0.45)\end{array}$ & $\begin{array}{l}2.70 \\
(0.95)\end{array}$ & $\begin{array}{l}1.78 \\
(1.09)\end{array}$ & $\begin{array}{l}2.83 \\
(0.75)\end{array}$ & $\begin{array}{l}2.46 \\
(1.21)\end{array}$ & 0.016 \\
\hline $\begin{array}{l}\text { 4. Complete personal business on } \\
\text { company time }\end{array}$ & $\begin{array}{l}2.91 \\
(1.15)\end{array}$ & $\begin{array}{l}2.44 \\
(1.19)\end{array}$ & $\begin{array}{l}2.29 \\
(1.04)\end{array}$ & $\begin{array}{l}2.42 \\
(1.09)\end{array}$ & $\begin{array}{l}1.60 \\
(1.34)\end{array}$ & $\begin{array}{l}2.90 \\
(1.20)\end{array}$ & $\begin{array}{l}2.56 \\
(1.01)\end{array}$ & $\begin{array}{l}3.67 \\
(0.82)\end{array}$ & $\begin{array}{l}2.38 \\
(0.92)\end{array}$ & 0.008 \\
\hline $\begin{array}{l}\text { 8. Do less than your share of } \\
\text { work in a group project }\end{array}$ & $\begin{array}{l}1.87 \\
(0.73)\end{array}$ & $\begin{array}{l}1.92 \\
(1.04)\end{array}$ & $\begin{array}{l}2.38 \\
(1.31)\end{array}$ & $\begin{array}{l}1.79 \\
(0.83)\end{array}$ & $\begin{array}{l}1.20 \\
(0.45)\end{array}$ & $\begin{array}{l}2.50 \\
(1.27)\end{array}$ & $\begin{array}{l}2.33 \\
(1.22)\end{array}$ & $\begin{array}{l}2.00 \\
(1.26)\end{array}$ & $\begin{array}{l}1.50 \\
(0.59)\end{array}$ & 0.007 \\
\hline $\begin{array}{l}\text { 21. Take money from the } \\
\text { company }\end{array}$ & $\begin{array}{l}1.45 \\
(0.65)\end{array}$ & $\begin{array}{l}1.60 \\
(0.91)\end{array}$ & $\begin{array}{l}1.71 \\
(1.04)\end{array}$ & $\begin{array}{l}1.41 \\
(0.71)\end{array}$ & $\begin{array}{l}1.00 \\
(0.00)\end{array}$ & $\begin{array}{l}1.60 \\
(1.08)\end{array}$ & $\begin{array}{l}1.11 \\
(0.33)\end{array}$ & $\begin{array}{l}2.17 \\
(1.47)\end{array}$ & $\begin{array}{l}1.75 \\
(1.07)\end{array}$ & 0.111 \\
\hline 25. Purchase items online & $\begin{array}{l}3.03 \\
(1.16)\end{array}$ & $\begin{array}{l}2.96 \\
(1.31)\end{array}$ & $\begin{array}{l}2.96 \\
(1.12)\end{array}$ & $\begin{array}{l}2.52 \\
(1.11)\end{array}$ & $\begin{array}{l}1.80 \\
(0.84)\end{array}$ & $\begin{array}{l}2.80 \\
(1.69)\end{array}$ & $\begin{array}{l}1.78 \\
(1.09)\end{array}$ & $\begin{array}{l}3.33 \\
(0.82)\end{array}$ & $\begin{array}{l}2.79 \\
(1.18)\end{array}$ & 0.017 \\
\hline
\end{tabular}

Table 3: Ethical Behaviour Belief Items and Religious Group Affiliation in the Subset of those Employed Part-time or Full-time

Note: CHRIS=Christian, JEW=Jewish, MUSL=Muslim, CATH=Catholic, HIN=Hindu, BUD=Buddhist, $\mathrm{AGN}=\mathrm{Agnostic}, \mathrm{ATH}=\mathrm{Atheist}, \mathrm{OTH}=\mathrm{Other}$

In the ANCOVA analysis adjusting for the religious observance level variable, the same pattern of significance occurred. In the ANCOVA analysis adjusting for numerous relevant covariates, the same pattern of significance or approaching significance occurred except for "take money from the company" which now had a p-value of 0.102 .

Post-hoc least significance difference tests for the 4 items with overall significance were as follows. For "take merchandise/equipment for one's own personal use," Hindu had the lowest mean and significantly differed from all religious groups except for Catholic and Agnostic. Agnostic had the next lowest mean and approached significance to differ from Muslim ( $\mathrm{p}=0.076)$, Buddhist $(\mathrm{p}=0.054)$, Atheist $(\mathrm{p}=0.054)$, and Other $(\mathrm{p}=0.094)$. Catholic had the third lowest mean and significantly differed from all religious groups except did not differ from Hindu and Agnostic as mentioned above and also did not differ from Jewish.

For "complete personal business on company time," Hindu had the lowest mean and significantly differed from Christian, Buddhist, and Atheist. Muslim had the second lowest mean and significantly differed from Christian and Atheist. Other had the third lowest mean and significantly differed from Christian and Atheist. Catholic had the fourth lowest mean and significantly differed from Christian and Atheist. Jewish had the fifth lowest mean and significantly differed from Atheist and also approached significance to differ from Christian $(\mathrm{p}=0.067)$. Agnostic had the sixth lowest mean and approached significance to differ from Atheist $(\mathrm{p}=0.057)$. 
For "do less than your share of work in a group project," Hindu had the lowest mean and significantly differed from Muslim, Buddhist, and Agnostic. Other had the second lowest mean and significantly differed from Muslim, Buddhist, and Agnostic and also approached significance to differ from Christian $(\mathrm{p}=0.087)$. Catholic had the third lowest mean and significantly differed from Muslim and Buddhist and also approached significance to differ from Agnostic $(p=0.091)$. Christian had the fourth lowest mean and significantly differed from Muslim and Buddhist. Jewish had the fifth lowest mean and approached significance to differ from Muslim ( $\mathrm{p}=0.080)$ and Buddhist $(\mathrm{p}=0.089)$.

For "purchase items online," Agnostic had the lowest mean and significantly differed or approached significance to differ from all the religious groups except for Hindu. Agnostic approached significance to differ from Catholic $(\mathrm{p}=0.074)$ and Buddhist $(\mathrm{p}=0.059)$. Hindu had the second lowest mean and significantly differed from Christian, Jewish, Muslim, Atheist and also approached significance to differ from Other $(\mathrm{p}=0.086)$. Catholic had the third lowest mean and significantly differed from Christian.

\section{Discussion}

We found that for our three analytic approaches (i.e., all items, overall significant items, and computer/Internet items) those who were Hindu or Catholic consistently had the lowest mean scores indicating beliefs in performing more ethical behaviour. Also, for our three analytic approaches, those who were Buddhist or Atheist consistently had the highest mean scores indicating beliefs in performing less ethical behaviour. In one analysis relating to computer/Internet items, those who were Jewish had the highest mean scores indicating beliefs in performing less ethical behaviour. All 27 items had some statistical difference, whether for the overall analysis or for the post-hoc tests.

It is challenging to directly compare the results from our study to the one other business ethics study that compares a proxy measure of religious affiliation based upon type of college attended. That study found Evangelicals to be more ethical that Catholics with regard to business ethics scenarios (Kennedy and Lawton 1998). Although in our study Catholics had beliefs in performing more ethical business behaviour than Christians, our study had the general category of Christian and it is possible that both Evangelicals and non-Evangelicals chose that category.

\section{Beliefs in Performing More Ethical Behaviour}

The beliefs in performing more ethical behaviour among Hindus can be understood by some aspects of the Hindu faith. The concept of the law of karma is that actions have an effect. If one does good deeds this generates good conditions for this individual while if one does bad deeds this generates bad conditions for this individual (Bilimoria 1994; Hunt et al. 1991; Rai 2005). It is possible that those who are Hindu believe that more ethical business behaviour will result in good future conditions for themselves and therefore they are likely to engage in more ethical business behaviour. Also, Hindus believe in many deities and gods. Hindu belief dictates that any god is a true god if it satisfies one's spiritual need (Hunt et al. 1991). As Hindus have many deities or gods, a Hindu can find a particular deity or god to identify with and practice more ethical behaviour as part of such observance. It may even be that a belief exists that one needs to answer to this particular favourite deity or god and be very careful about business ethical behaviour.

The beliefs in performing more ethical behaviour among Catholics can be understood by aspects of the Catholic faith. The Bible, Councils of the Church, Papal decrees, and Code of Canon Law dictate the approach and behaviour for Catholics. This extensive body of religious law and philosophical teachings guides and provides an approach for Catholics to addressing almost any moral or ethical dilemma (Hunt et al. 1991). Catholics with their many rules and rituals integrate and incorporate these rules and rituals into their more ethical behaviour. They also may be more ethical due to a belief that their behaviours are answerable to God and they want to avoid sin. 


\section{Beliefs in Performing Less Ethical Behaviour}

The beliefs in performing less ethical behaviour of Atheists can be suggested by the lack of a religious basis or tradition of ethics. Atheism by definition is the lack of belief in God or practice of organized religion. As there is no formal religious ethical code to follow, it could be that for those gray areas that are not specifically dictated in secular law, those who are Atheist may tend to be less ethical since they choose a person-centred approach and perform the behaviours that favour their personal interest as the main interest.

The beliefs for performing less ethical behaviour among Buddhists are challenging to understand. In both the Theravada and Mahayana approaches to Buddhism there is a rich tradition of ethics which followers can practice (Hunt et al. 1991). One can classify Buddhism as an ethics system of virtues and vices where there are three groups of virtues that include 1) conscientiousness, 2) benevolence and 3) self-restraint (De Silva 1994). However, culture can relate to differences in the business ethics approach used (Rashid and Ibrahim 2008). Among all the religious groups in this study, those who were Buddhist had the greatest percentage at $75 \%$ of those who were not born in the United States. It is possible that it is not the practice of Buddhism per se that is associated with beliefs in performing less ethical behaviour but rather a non United States business ethics approach that differs from the typical business cultural ethical norms practiced in the United States.

There were beliefs in performing less ethical behaviour for computer/Internet topics among those Jewish. The Jewish faith has an extensive ethical tradition based upon the Bible, Talmud, and Shulkhan Aruch (Fogel and Friedman 2008; Friedman 1985). This rich code of religious practices is constantly interpreted for day-to-day practice (Hunt et al. 1991). Unlike other faiths, there is often no central authority in Judaism. Jews often choose which leader or rabbi to follow. Many Jews do not necessarily follow any particular leader or rabbi and instead make their own decisions when interpreting contemporary issues that are not directly discussed in the Bible, Talmud, or Shulkhan Aruch. It could be that Jews are considering less strict interpretations for these contemporary computer/Internet topics. After all, it may be easy to rationalize and believe that one will always be careful and strictly follow all the numerous laws of Judaism. However, since the Bible, Talmud, and Shulkhan Aruch do not specifically address computer/Internet topics perhaps there is some loophole to not be so strict about certain business ethical topics related to computer/Internet topics.

\section{Employment Experience}

Some suggest that students have different approaches to business ethics than those employed due to the lack of practical experience with ethical issues (Ibrahim et al. 2008). Our study differed from this suggestion. In our study among the subset of those employed part-time or full-time similar patterns were seen as among the whole sample. This included similar overall significance patterns and also similar beliefs in performing ethical behaviour patterns.

\section{Study Limitations and Future Directions}

There are a number of limitations. First, some of the religious groups had a small number of individuals. Second, many religions have different approaches for that religion (e.g., for Muslims there is Sunni and Shiite, for Jewish there is Orthodox, Conservative, and Reform) and this was not measured. Third, although we inquired about religious observance level, we did not inquire specifically about an individual's ethical knowledge about the individual's particular religious classification. Fourth, this study is among individuals from New York City, a cosmopolitan large city in the United States and may not generalize to other regions of the United States. Future research should include larger samples from each religious category, measurement with sufficiently large sample size for the different religious approaches practiced by each religion, ethical knowledge assessment for each particular religious classification, and individuals from multiple regions of the United States. 


\section{Conclusions}

In conclusion, college students who are Hindu and Catholic have beliefs consistent with the most ethical approach for a number of business ethics behaviours. Also, college students who are Atheist and Buddhist have beliefs consistent with the least ethical approach for a number of business ethics behaviours. College students who are Jewish have beliefs consistent with the least ethical approach for computer and Internet behaviours. Managers at businesses who supervise young adults who recently graduated from college can be better aware of the possible ethical concerns facing those of different religions. They can mentor these individuals beginning their careers to adapt ethical behaviours appropriate for their specific business setting.

\section{Acknowledgements}

Portions of this manuscript were presented at the $15^{\text {th }}$ Annual International Conference Promoting Business Ethics, New York, NY, October 22-24, 2008, and the 20th Greater New York Conference on Behavioural Research, New York, NY, November 14, 2008.

\section{References}

American Management Association (2006) "The ethical enterprise" Available at http://www.amanet.org/research/pdfs/HREthicsSurvey06.pdf . Accessed December 12, 2008.

Bilimoria, P. (1994) "Indian Ethics" in P. Singer (ed.), A Companion to Ethics, Blackwell, Cambridge, MA, pp. 43-57.

Conroy, S. J. and T. L. N Emerson (2004) "Business Ethics and Religion: Religiosity As A Predictor of Ethical Awareness Among Students" Journal of Business Ethics 50(4): 383-396.

De Silva, P. (1994), "Buddhist Ethics" in P. Singer (ed.), A Companion to Ethics, Blackwell, Cambridge, MA, pp. 58-68.

Ethics Resource Center (2007) "National Business Ethics Survey" Ethics Resource Center, Arlington, VA. Available at http://www.ethics.org/research/NBESOffers.asp . Accessed December 12, 2008.

Fogel, J., and H. H. Friedman (2008) "Conflict of Interest and the Talmud" Journal of Business Ethics 78: $237-246$.

Friedman, H. H. (1985) "Ethical Behaviour in Business" A Hierarchical Approach from the Talmud" Journal of Business Ethics 4: 117-129.

Ghorpade, J., J. Lackritz and G. Singh (2006) "Correlates of the Protestant Ethic of Hard Work: Results from a Diverse Ethno-religious Sample” Journal of Applied Social Psychology 36(10): 2449-2473.

Graafland, J., M. Kaptein and C. Mazereeuw-van der Duijn Schouten (2006) "Business Dilemmas and Religious Belief: An Explorative Study Among Dutch Executives" Journal of Business Ethics 66(1): 53-70.

Greenberg, J. (1990) "Employee Theft as a Reaction to Underpayment Inequity: The Hidden Cost of Pay Cuts" Journal of Applied Psychology 75(5): 561-568.

Greenberg, J. (1993) "Stealing in the Name of Justice: Informational and Interpersonal Moderators of Theft Reactions to Underpayment Inequity" Organizational Behaviour \& Human Decision Processes 54(1): 81-103.

Hunt, A. D., M. T. Crotty and R. B. Crotty (1991) Ethics of World Religions, revised edition, Greenhaven, San Diego, CA.

Hunt, S. D., S. J. Vitell (2006) "The General Theory of Marketing Ethics: A Revision and Three Questions" Journal of Macromarketing 26(2): 143-153.

Ibrahim, N., D. Howard and J. Angelidis (2008) "The Relationship Between Religiousness and Corporate Social Responsibility Orientation: Are There Differences Between Business Managers and Students?" Journal of Business Ethics 78(1/2): 165-174.

Jones, J. R., A. N. Christopher, P. Marek and D. Reinhart (2005) "Student's Perceptions of Questionable Workplace Behaviours: The Effects of Perceiver and Actor Attributes" Individual Differences Research 3(1): 14-26. 
Jones, G. E., and M. J. Kavanagh (1996) "An Experimental Examination of the Effects of Individual and Situational Factors on Unethical Behavioural Intentions in the Workplace" Journal of Business Ethics 15(5): 511-523.

Kennedy, E. J. and L. Lawton (1998) "Religiousness and Business Ethics" Journal of Business Ethics 17(2): 163-175.

McCabe, C. A., R. Ingram, and M. C. Dato-on (2006) "The Business of Ethics and Gender" Journal of Business Ethics 64(2): 101-116.

McDonald, G. M., and C. K. Pak (1997) "Ethical Perceptions of Expatriate and Local Managers in Hong Kong” Journal of Business Ethics 16(15): 1605-1623.

Muhamad, R. (2009). "Religiosity, Ethical Judgments and Malaysian Muslim Students" Journal of Business Systems, Governance and Ethics 4(1). Available at http://www.jbsge.vu.edu.au/issues/vol04no1/Muhamad.pdf . Accessed February 25, 2010.

Nonis, S., and C. O. Swift (2001) "An Examination of the Relationship Between Academic Dishonesty and Workplace Dishonesty: A Multi-campus Investigation” Journal of Education for Business 77(2): 69-77.

Rai, H. (2005) "The Role of Hinduism in Global India and her Business Ethics" in N. Capaldi (ed.), Business and Religion: A Clash of Civilizations, M \& M Scrivener, Salem, MA, pp. 379-389.

Rashid, M. Z. and S. Ibrahim (2008) "The Effect of Culture and Religiosity on Business Ethics: A Cross-cultural Comparison" Journal of Business Ethics 82: 907-917.

SPSS (2008). Version 16.0, SPSS, Chicago, IL. 
